[Original]

\title{
Identifying Activity Limitation and Decline in Quality of Life in Patients with Knee Osteoarthritis Who Are Scheduled to Undergo Total Knee Arthroplasty
}

\author{
Tetsuya Amano ${ }^{*}$, Shigeharu TANAKA ${ }^{2}$ and Hideyuki Ito $^{3}$ \\ ${ }^{1}$ Department of Physical Therapy, Faculty of Health and Medical Sciences, Tokoha University. Kita-ku, Hamamatsu \\ 431-2102, Japan \\ 2 Division of Physical Therapy, School of Rehabilitation, Faculty of Health and Social Services, Kanagawa University \\ of Human Services. Yokosuka 238-8522, Japan \\ ${ }^{3}$ Major of Physical Therapy, Department of Rehabilitation, Faculty of Wakayama Health Care Sciences, Takarazuka \\ University of Medical and Health Care. Wakayama 640-8392, Japan
}

\begin{abstract}
This study examines factors associated with activity limitation and the decline in quality of life in patients with knee osteoarthritis (OA), and to determine standard values that would be useful for understanding the disease state. We assessed individual factors, the modified version of the Self-Rating Frenchay Activities Index (SR-FAI), and the Japanese Knee Osteoarthritis Measure (JKOM) of 661 participants. Multiple regression analyses were used to examine the relationship between dependent and explanatory variables. Standard values were calculated after stratifying based on factors related to each dependent variable through multiple regression analyses. Among the participants aged 60-74/75-89 years, the standard values for the modified SR-FAI were 23/19 and 20/18 points for K-L grades III and IV in the males, and $29 / 23$ and $26 / 21$ points in the females, respectively. The standard values for the JKOM were 35/49 and 45/59 points for K-L grades III and IV in the males and 41/52 and 47/60 points in the females, respectively. These standard values are a useful index for interpreting the degree of activity limitation and satisfaction reduction in patients with knee OA, and could help in clinical decision making for healthcare providers.
\end{abstract}

Keywords : knee osteoarthritis, standard value, modified version of Self-Rating Frenchay Activities Index, Japanese Knee Osteoarthritis Measure, physical and rehabilitation medicine.

(Received October 22, 2020, accepted November 27, 2020)

\section{Introduction}

Knee osteoarthritis $(\mathrm{OA})$ is the most common articular disease that limits the activity of older adults, and an increase in the number of patients with knee $\mathrm{OA}$ is expected in developed countries [1]. Previous studies have reported that knee OA is a risk factor for falls and results in limitations of functional ability and decline in quality of life (QOL) [2,3], and is a degenera- tive disease with symptoms that worsen over time [4]. Conservative therapy of knee OA is the first choice for treatment, but patients with worse symptoms are recommended to undergo surgery. Hence, it is important to interpret the limitations of activities of daily living (ADL) and the decline in QOL in patients with knee OA adequately, as well as to provide treatment according to the disease state of the individual.

Total knee arthroplasty (TKA) is the most com-

*Corresponding Author: Tetsuya Amano, Ph.D., Department of Physical Therapy, Faculty of Health and Medical Sciences, Tokoha University, 1230 Miyakoda, Kita-ku, Hamamatsu Shizuoka 431-2102, Japan, Tel: +81-53-428-1211, Fax: +81-53-428-1202, E-mail: tamano@hm.tokoha-u.ac.jp 
monly performed surgery for knee OA, as it is an effective treatment [5]. Previous studies have reported that TKA for knee OA is usually performed in cases of severe $\mathrm{OA}$ with joint destruction and contracture, and over 60 years of age $[6,7]$. There is no standard for determining the appropriateness of TKA, however, because there are few useful indicators available for judging the limitations of conservative therapy. Disease-based stratified information also contributes to a better understanding of the patients' condition. For these reasons, we think it is necessary to clarify the degree of preoperative activity limitation and preoperative decline in QOL in patients who are scheduled to undergo TKA.

To accurately identify the disease state of patients with a wide variety of clinical features, we need to clarify the values that could generalize the degree of limitation in ADL and decline in QOL in patients with knee OA. The representative index for evaluating ADL is the Functional Independence Measure (FIM), which is widely used internationally, including in Japan $[8,9]$. It is important to examine the reactivity of the evaluation scales as well as ceiling and floor effects in the targeted disease in order to appropriately understand the activity limitation of individuals. In our previous study we investigated the total scores of preoperative FIM in patients with knee OA who were scheduled to undergo TKA through minimally invasive surgery and found that the mean total scores of preoperative FIM was $120.8 \pm 4.4$ points, which was a ceiling effect, indicating that the use of FIM to properly evaluate activity disorders in patients with knee OA is limited [10].

The FIM includes basic activities in daily life such as eating, bathing, dressing, toileting, transfer, walking, and climbing stairs, while the modified version of Self-Rating Frenchay Activities Index (SR-FAI) assesses instrumental activities in daily life such as preparing main meals, washing up, washing clothes, housework, local shopping, and travel outings [11]. Considering the ceiling effect of FIM in patients with knee OA, we should determine the degree of activity limitation using the modified version of SRFAI, which has a broader use than FIM. Moreover, given that the Japanese Knee Osteoarthritis Measure (JKOM) is widely used in Japan as a QOL scale in pa- tients with knee OA $[12,13]$, we should use the JKOM to identify the degree of decline in QOL in Japanese patients with knee OA.

The purpose of this study was to examine factors associated with activity limitation and decline in quality of life in patients with knee OA who are scheduled to undergo TKA, and to determine the standard values which would be useful for understanding the disease state.

\section{Methods}

\section{Study design}

This study had a cross-sectional design. All the participants were informed of the purpose of this study and provided written informed consent. This study was approved by the Research Ethics Committee of Tokoha University (approval no. R-2018-504H).

\section{Setting}

Participant recruitment and data collection were performed between July 2013 and December 2018 at 15 general hospitals through a general recruitment.

\section{Participants}

The inclusion criteria were as follows: (1) patients who were scheduled to undergo TKA, (2) aged between 60 and 89 years, and (3) had a diagnosis of OA with Kellgren-Lawrence (K-L) grade III or IV of the surgical side. The exclusion criteria were as follows: (1) patients who had significant functional joint disorders other than the knee OA, (2) patients with neurological impairment such as motor paralysis, (3) patients with dementia, and (4) patients with mental disorders such as depression.

\section{Variables}

This study assessed the following individual factors: sex, age, body mass index (BMI), K-L grade, affected side, surgical history, and knee joint pain. We also measured FIM as the ADL scale, the modified version of SR-FAI as the instrumental activities of daily living (IADL) scale, and JKOM as the QOL scale. Affected side was whether the patient was diagnosed with unilateral knee OA or bilateral knee OA, and the patients with a history of contralateral TKA were classified as 
bilateral knee OA. History of surgery was examined prior to history of contralateral TKA. Knee joint pain was evaluated using a numerical rating scale (NRS) that ranged from 0 (no pain) to 10 (worst possible pain) [14].

The FIM is an ADL scale that includes self-care, sphincter control, transfers, locomotion, communication, and social cognition [15]. It is a commonly used ADL scale that consists of 18 items, and its reliability has been shown in a previous study. The FIM score evaluates each item according to seven levels (1 to 7 points), with the total score ranging from 18 points to 126 points. A higher FIM score indicates better ADL.

The modified version of SR-FAI is an IADL scale that includes housework, local shopping, walking outside, travel outings, actively pursuing a hobby, and gainful work $[16,17]$. It is an IADL scale that consists of 15 items, and its reliability and validity have been shown in a previous study. The modified version of SR-FAI score evaluates each item according to four levels ( 0 to 3 points), with the total score ranging from 0 points (inactive) to 45 points (highly active).

The JKOM is a quality of life scale that includes pain and stiffness in the knees, condition in daily life, general activities, and health conditions [18]. It is a specific QOL scale for patients with knee OA that consists of 25 items, and its reliability and validity have been examined in a previous study. The JKOM score evaluates each item according to five levels (0 to 4 points), with the total score ranging from 0 points to 100 points. A lower JKOM score indicates better QOL. Preoperative evaluation of the individual factors and these scales were performed at the time of hospitalization.

\section{Statistical analysis}

First, to examine the criterion-related validity of the modified version of SR-FAI in patients with knee OA, we determined its correlation with FIM and JKOM using Pearson's correlation coefficient. Second, we established two models using the total scores of the modified version of SR-FAI and JKOM as dependent variables. Multiple regression analyses were utilized to examine the relationship between the dependent and explanatory variables. The explanatory variables were sex, age, BMI, K-L grade, affected side, surgical history, and knee joint pain on NRS. The explanatory variables were entered into the multiple regression models by the stepwise method. Qualitative data were converted to dummy variables as follows: sex (male $=1$, female $=0), \mathrm{K}-\mathrm{L}$ grade $($ grade $\mathrm{III}=1$, grade $\mathrm{IV}=$ 0 ), affected side (unilateral knee $\mathrm{OA}=1$, bilateral knee $\mathrm{OA}=0$ ), and surgical history (no $=1$, yes $=0$ ). Other variables (age, BMI, and NRS) were treated as quantitative data. Finally, the standard values of the modified version of SR-FAI and the JKOM were calculated after stratifying based on significant related factors of each dependent variable. The software used to analyze the data was SPSS 22.0 (IBM Corp., Tokyo, Japan), and the significance level was set at $P=0.05$.

\section{Results}

A total of 661 patients (532 females, 129 males; mean age: 74.9 [standard deviation (SD) 6.5] years) participated in this study. The characteristics of the participants are summarized in Table 1. The mean FIM in patients with knee OA was $121.5 \pm 5.5$ points, and the mean +1 SD exceeded 126 points, which was the full score. Thus, we judged that FIM had a ceiling effect problem in evaluating the activity level in patients with knee OA. The modified version of SR-FAI correlated significantly with the FIM $(\mathrm{r}=0.379, P<0.001)$ and the JKOM ( $\mathrm{r}=-0.263, P<0.001)$, but the Pearson's correlation coefficient showed low values. In particular, the modified version of SR-FAI was shown to have criterion-related validity and discriminant validity with the FIM and the JKOM.

In the multiple regression analysis, age $(\beta=-0.222$; $P<0.001)$, sex $(\beta=-0.164 ; P<0.001)$, and $\mathrm{K}-\mathrm{L}$ grade $(\beta=0.103 ; P=0.010)$ correlated statistically with the modified version of SR-FAI (Table 2). Specifically, patients who were young, female, and had a K-L grade of III obtained high total scores in the modified version of SR-FAI, indicating that they were highly active. Age $(\beta=0.220 ; P<0.001), \mathrm{K}-\mathrm{L}$ grade $(\beta=-0.127$; $P=0.001)$, and sex $(\beta=-0.121 ; P=0.002)$ also correlated statistically with the JKOM (Table 2). Thus, patients who were younger, had a K-L grade of III, and were male obtained low total JKOM scores, indicating high levels of QOL.

Consequently, the standard values of the modified version of SR-FAI and the JKOM in patients with knee OA were derived after stratifying according to sex, age, 
Table 1. Characteristics of the participants

\begin{tabular}{|c|c|c|c|}
\hline \multicolumn{3}{|c|}{ Variable } & Value \\
\hline \multirow{11}{*}{$\begin{array}{l}\text { Individual } \\
\text { factors }\end{array}$} & Sex & Male & $129(19.5)$ \\
\hline & & Female & $532(80.5)$ \\
\hline & Age (years) & & $74.9 \pm 6.5$ \\
\hline & BMI $\left(\mathrm{kg} / \mathrm{m}^{2}\right)$ & & $25.7 \pm 3.9$ \\
\hline & K-L grade & Grade III & $263(39.8)$ \\
\hline & & Grade IV & $398(60.2)$ \\
\hline & Affected side & Unilateral knee OA & $131(19.8)$ \\
\hline & & Bilateral knee OA & $530(80.2)$ \\
\hline & Surgical history & No & $462(69.9)$ \\
\hline & & Yes & $199(30.1)$ \\
\hline & NRS (points) & & $3.1 \pm 3.1$ \\
\hline $\mathrm{ADL}$ & FIM (points) & & $121.5 \pm 5.5$ \\
\hline IADL & Modified versior & of SR-FAI (points) & $23.2 \pm 9.0$ \\
\hline QOL & JKOM (points) & & $50.9 \pm 20.1$ \\
\hline
\end{tabular}

Values are presented as mean \pm standard deviation or $\mathrm{n}(\%)$. BMI: body mass index, K-L grade: Kellgren-Lawrence grade, OA: osteoarthritis, NRS: numerical rating scale, ADL: activities of daily living, FIM: Functional Independence Measure, IADL: instrumental activities of daily living, SR-FAI: Self-Rating Frenchay Activities Index, QOL: quality of life, JKOM: Japanese Knee Osteoarthritis Measure

Table 2. Related factors of modified version of SR-FAI and JKOM

\begin{tabular}{llllllll}
\hline Model & Variable & $\beta$ & $P$-value & B & \multicolumn{2}{c}{$\begin{array}{c}95 \% \\
\text { Lower }\end{array}$} \\
\hline $\begin{array}{l}\text { Modified } \\
\text { version of } \\
\text { SR-FAI }\end{array}$ & Sex & -0.164 & $<0.001$ & -3.720 & -5.491 & -1.949 \\
& K-L grade & 0.103 & 0.010 & 1.900 & 0.464 & 3.335 \\
& Age & 0.220 & $<0.001$ & 0.680 & 0.446 & 0.914 \\
JKOM & K-L grade & -0.127 & 0.001 & -5.148 & -8.215 & -2.080 \\
& Sex & -0.121 & 0.002 & -6.106 & -9.939 & -2.272 \\
\hline
\end{tabular}

Modified SR-FAI model: $\mathrm{R}=0.298, \mathrm{R}^{2}=0.089$, adjusted $\mathrm{R}^{2}=$ $0.084, P<0.001$. JKOM model: $\mathrm{R}=0.286, \mathrm{R}^{2}=0.082$, adjusted $\mathrm{R}^{2}=0.077, P<0.001$. $\beta$ : standardized partial regression coefficient, B: partial regression coefficient, CI: confidence interval, SR-FAI: Self-Rating Frenchay Activities Index, JKOM: Japanese Knee Osteoarthritis Measure, K-L grade: Kellgren-Lawrence grade and K-L grade. The standard value of this study was shown using the median, because each data after stratifying did not follow a normal distribution. Among the patients aged $60^{-74 / 75-89}$ years, the standard values for the modified version of SR-FAI were 23/19 and 20/18 points for K-L grades III and IV in the males, and 29/23 and 26/21 points in the females, respectively (Table 3). Additionally, the standard values for the JKOM among patients aged 60-74/75-89 years were $35 / 49$ and 45/59 points for K-L grades III and IV in the males and $41 / 52$ and $47 / 60$ points in the females, respectively (Table 4).

\section{Discussion}

In the present study, we determined the standard values that were useful for understanding the disease state of knee OA patients who were scheduled to undergo TKA by examining the actual state of activity limitation and decline in QOL. This study revealed that the

Table 3. Standard values for total scores of modified version of SR-FAI

\begin{tabular}{llcccc}
\hline K-L grade & Sex & n & $60-74$ years & n & $75-89$ years \\
\hline Grade III & Male & 26 & $23(19-29)$ & 30 & $19(14-27)$ \\
& Female & 99 & $29(25-34)$ & 108 & $23(19-28)$ \\
Grade IV & Male & 35 & $20(13-27)$ & 38 & $18(11-26)$ \\
& Female & 135 & $26(20-32)$ & 190 & $21(15-27)$ \\
\hline
\end{tabular}

Values are presented as median (interquartile range). Units are in points. SR-FAI: Self-Rating Frenchay Activities Index, K-L grade: Kellgren-Lawrence grade

Table 4. Standard values for total scores of JKOM

\begin{tabular}{llcccc}
\hline K-L grade & Sex & $n$ & $60-74$ years & $n$ & $75-89$ years \\
\hline Grade III & Male & 26 & $35(28-44)$ & 30 & $49(36-64)$ \\
& Female & 99 & $41(29-58)$ & 108 & $52(37-70)$ \\
Grade IV & Male & 35 & $45(33-56)$ & 38 & $59(37-69)$ \\
& Female & 135 & $47(35-61)$ & 190 & $60(43-72)$ \\
\hline
\end{tabular}

Values are presented as median (interquartile range). Units are in points. JKOM: Japanese Knee Osteoarthritis Measure, K-L grade: Kellgren-Lawrence grade 
male patients, those aged 75-89 years, and those with $\mathrm{K}-\mathrm{L}$ grade of IV had low total scores in the modified version of SR-FAI, and that the female patients, those aged 75-89 years, and those with K-L grade of IV had high total scores of JKOM. Consequently, the standard values of the modified version of SR-FAI and the JKOM were calculated after stratifying according to these related factors. The standard values of the modified version of SR-FAI and the JKOM in this study were the total scores of patients with knee OA before TKA, and can be used to interpret the degree of activity limitation and decline in QOL in these patients. These standard values could help in clinical decision making for healthcare providers.

A previous study reported that females aged 5579 years living at home had higher total scores on the modified version of SR-FAI than males, and were more active than males [17]. The results of the present study showed that the total score on the modified version of SR-FAI was higher in females than in males, even in patients with knee OA before TKA, and the activity of females was better than that of males. Furthermore, as far as we know, there is no study to have ever examined sex differences in the JKOM. On the other hand, a previous study reported a significant difference between male and female patients with knee $\mathrm{OA}$ in Western Ontario and McMaster Universities Osteoarthritis Index (WOMAC) scores and Medical Outcomes Study 36-Item Short-Form Health Survey (SF-36) scores; that is, female patients had significantly worse symptoms than male patients [19]. The present study showed that the total score of JKOM was higher in females than in males, even in patients with knee OA before TKA, and the QOL of females was worse than that of males. Given that these standard values were created from many samples, we consider that these standard values stratified by related factors can be useful indices for interpreting the degree of activity limitation and decline in QOL in patients with knee OA.

Based on the results of the present study, the standard values for the modified version of SR-FAI among patients aged 60-74/75-89 years were 23/19 and 20/18 points for K-L grades III and IV, respectively, in the males, and $29 / 23$ and $26 / 21$ points, respectively, in the females. A previous case-comparison study reported that the mean scores on the modified version of SR-FAI in community-dwelling older adults (mean age, 64.4 years for male; mean age, 65.1 years for female) with stroke and in community-dwelling healthy elderly (age 55-74 years) were 12.0/23.1 points (males/females) and 26.8/34.6 points (males/females), respectively [20]. Given that the activity limitation in patients with knee OA is different between healthy older adults and patients with stroke, generalizing the standard values obtained in the previous studies is not ideal. Thus, the standard value of the modified version of SR-FAI in the present study could be a source of valuable information to determine the degree of activity limitation in patients with knee OA.

In the results of this study, the standard values for the JKOM among patients aged 60-74/75-89 years were $35 / 49$ and $45 / 59$ points for K-L grades III and IV, respectively, in the males, and 41/52 and 47/60 points, respectively, in the females. A previous crosssectional observational study examined the median of the JKOM total score in patients with knee OA from community orthopedic clinics and showed that the total scores of the JKOM decreased as the number of daily steps increased (JKOM total scores: 25 points for patients with $<2,500$ steps/day and 12 points for patients with $>7,500$ steps/day) [21]. The standard values of JKOM in this study were higher than those in that previous study. In other words, the level of QOL in patients with knee OA who were scheduled to undergo TKA was worse than that in community-dwelling patients. Thus, the standard values of the JKOM in this study can be useful for interpreting the degree of decline in QOL in patients with severe knee OA.

This study had some limitations. First, as we excluded patients who had been diagnosed with multiple-joint OA such as hip OA and spine OA, it is unclear whether these standard values can be used in a population with OA in multiple joints. Second, these standard values cannot be used as indices for judging the application of treatment, because this study was not a comparison with a control group. Third, medical information such as data on the onset of knee OA, period of commencement of rehabilitation, and contents of medical treatment, which might also have affected the results of our present study, was not obtained. Future studies should include this additional information. 


\section{Conclusion}

Given that the standard values of the modified version of SR-FAI and the JKOM used in this study were created from many samples, these values could be useful indices for interpreting the degree of activity limitation and decline in QOL in patients with knee OA. Accordingly, our findings suggest that these standard values could help in clinical decision making for healthcare providers.

\section{Acknowledgments}

We wish to thank the physical therapists at all the 15 hospitals that participated in this study. This work was supported by JSPS KAKENHI Grant number JP25750245.

\section{Conflicts of Interest}

The authors declare that there is no conflict of interest.

\section{References}

1. Inacio MCS, Paxton EW, Graves SE, Namba RS \& Nemes S (2017): Projected increase in total knee arthroplasty in the United States - an alternative projection model. Osteoarthritis Cartilage 25(11): 1797-1803

2. Muraki S, Akune T, Oka H et al (2013): Physical performance, bone and joint diseases, and incidence of falls in Japanese men and women: a longitudinal cohort study. Osteoporos Int 24(2): 459-466

3. Alkan BM, Fidan F, Tosun A \& Ardicoglu O (2014): Quality of life and self-reported disability in patients with knee osteoarthritis. Mod Rheumatol 24(1): 166-171

4. Yoshimura N, Muraki S, Oka H et al (2009): Prevalence of knee osteoarthritis, lumbar spondylosis, and osteoporosis in Japanese men and women: the research on osteoarthritis/osteoporosis against disability study. J Bone Miner Metab 27(5): 620-628

5. Zhang W, Nuki G, Moskowitz RW et al (2010): OARSI recommendations for the management of hip and knee osteoarthritis: part III: Changes in evidence following systematic cumulative update of research published through January 2009. Osteoarthritis Cartilage 18(4): 476-499
6. Riis A, Rathleff MS, Jensen MB \& Simonsen O (2014): Low grading of the severity of knee osteoarthritis preoperatively is associated with a lower functional level after total knee replacement: a prospective cohort study with 12 months' follow-up. Bone Joint J 96-B(11): 1498-1502

7. Thomsen MG, Husted H, Otte KS, Orsnes T \& Troelsen A (2012): Indications for knee arthroplasty have remained consistent over time. Dan Med J 59(8): A4492

8. Prodinger B, O'Connor RJ, Stucki G \& Tennant A(2017): Establishing score equivalence of the Functional Independence Measure motor scale and the Barthel Index, utilising the International Classification of Functioning, Disability and Health and Rasch measurement theory. J Rehabil Med 49(5): 416-422

9. Kidd D, Stewart G, Baldry J et al (1995): The Functional Independence Measure: a comparative validity and reliability study. Disabil Rehabil 17(1): 10-14

10. Amano T, Tamari K, Tanaka S et al (2016): Factors for assessing the effectiveness of early rehabilitation after minimally invasive total knee arthroplasty: A prospective cohort study. PLoS One 11(7): e0159172

11. Suenaga H, Miyanaga K, Chisaka H, Kawazu T \& Hachisuka K (2000): Modified version of the SelfRating Frenchay Activities Index and its reliability and validity. JJOMT 48(1):55-60 (in Japanese)

12. Sugita T, Kikuchi Y, Aizawa T, Sasaki A, Miyatake N \& Maeda I (2015): Quality of life after bilateral total knee arthroplasty determined by a 3-year longitudinal evaluation using the Japanese knee osteoarthritis measure. J Orthop Sci 20(1): 137-142

13. Sugita T, Miyatake N, Aizawa T, Sasaki A, Kamimura M \& Takahashi A (2019): Quality of life after staged bilateral total knee arthroplasty: a minimum five-year follow-up study of seventy-eight patients. Int Orthop 43(10): 2309-2314

14. Williamson A \& Hoggart B (2005): Pain: a review of three commonly used pain rating scales. J Clin Nurs 14(7): 798-804

15. Ottenbacher KJ, Hsu Y, Granger CV \& Fiedler RC (1996): The reliability of the functional independence measure: a quantitative review. Arch Phys Med Rehabil 77(12): 1226-1232

16. Schuling J, de Haan R, Limburg $M$ \& Groenier $\mathrm{KH}$ (1993): The Frenchay Activities Index. Assessment of functional status in stroke patients. Stroke 24(8): 
$1173-1177$

17. Hachisuka K, Saeki S, Tsutsui Y et al (1999): Genderrelated differences in scores of the Barthel Index and Frenchay activities index in randomly sampled elderly persons living at home in Japan. J Clin Epidemiol 52(11): 1089-1094

18. Akai M, Doi T, Fujino K, Iwaya T, Kurosawa H \& Nasu $\mathrm{T}$ (2005): An outcome measure for Japanese people with knee osteoarthritis. J Rheumatol 32(8): 1524-1532

19. Elbaz A, Debbi EM, Segal G et al (2011): Sex and body mass index correlate with Western Ontario and McMaster Universities Osteoarthritis Index and quality of life scores in knee osteoarthritis. Arch Phys Med Rehabil
92(10): 1618-1623

20. Hachisuka K, Tsutsui Y, Furusawa K \& Ogata H (1998): Gender differences in disability and lifestyle among community-dwelling elderly stroke patients in Kitakyushu, Japan. Arch Phys Med Rehabil 79(8): 998-1002

21. Iijima H, Fukutani N, Isho T et al (2017): Relationship between pedometer-based physical activity and physical function in patients with osteoarthritis of the knee: A cross-sectional study. Arch Phys Med Rehabil 98(7): 1382-1388

J UOEH $43(1): 33-39(2021)$ 